\title{
Development of water saving technology for water supply system of industrial enterprises
}

\author{
Juriy Lavirko ${ }^{1[0000-0002-1451-2176]}$, Eduard Akhmetov ${ }^{2[0000-0003-0179-4263]}$, Rimma \\ Akhmetova $^{2[0000-0003-3137-7727] *}$, and Natalia Bikeeva ${ }^{20000-0002-2148-0504]}$ \\ ${ }^{1}$ Kazan State University of Architecture and Engineering, 420043, Zelenaya str., Kazan, Russia \\ ${ }^{2}$ Kazan State Power Engineering University, 420066, Krasnoselskaya st., Kazan, Russia
}

\begin{abstract}
The object of the study is the water supply and sanitation system of an industrial enterprise. Currently, many enterprises do not use the treated industrial waste water in the water supply cycle. Therefore, the use of treated wastewater is of practical importance. The scheme of thermal treatment of waste water for obtaining pure condensate by evaporation is proposed. The main results of the study are the technical solution for the development of the enterprise's wastewater treatment complex. Pure condensate is obtained in an instant boiling evaporator. The research method is based on the calculation of energy costs in wastewater treatment for different types of waste water. The results of the study allow us to determine the optimal set of energy equipment for obtaining pure condensate and its further use. The specific practical significance lies in the development of a technical solution that helps the treatment of wastewater and the reduction of emissions into the water basin.
\end{abstract}

Keywords: water supply, wastewater treatment, heat treatment, instantboiling evaporator.

\section{Introduction}

According to the state policy in the field of environmental development of the Russian Federation for the period of up to 2030, the strategic goal is defined as the solution of social and economic problems, providing an environmentally-oriented economic growth, preservation of favorable environment [1-5]. Implementation and use of low-waste and resource-saving technologies and equipment to solve the problem to ensure environmentally sound waste management is required [6-11]. One of the most important issues of environmental protection is to protect the drainage basin from contamination [1113]. Important measures for the protection of water sources include industrial wastewater purification and further use of wastewater for industrial water supply [14-15]. The article describes a variant of a wastewater treatment enterprise with the subsequent return of treated water into the water system.

Water-use efficiency in industry is estimated by the percentage of water used in the reverse system $P_{r e v}$, rational use factor of water taken from the source $K_{r u}$ and the percentage of water loss $P_{\text {loss }}$ :

\footnotetext{
*Corresponding author: ahmetova_rv@bk.ru
} 


$$
\begin{gathered}
P_{r e v}=\frac{Q_{r e v}}{Q_{s}+Q_{r e v}+Q_{m}} \cdot 100 \Rightarrow 100 \% ; \\
K_{u}=\frac{Q_{s}+Q_{m}+Q_{t f}}{Q_{s}+Q_{m}} \leq 1 ; \\
K_{u}=\frac{Q_{s}+Q_{m}-Q_{t f}}{Q_{s}+Q_{m}} \leq 1 \\
P_{\text {loss }}=\frac{Q_{s}+Q_{m}-Q_{t f}}{Q_{s}+Q_{\text {rev }}+Q_{m}} \cdot 100 \Rightarrow 0 ;
\end{gathered}
$$

where $Q_{S}$ - amount of water taken from the source, $Q_{r e v}$ - the amount of water used in the back line of the system, $Q_{m}$ - the amount of water coming from the raw materials, $Q_{\text {rev }}-$ amount of water supplied to treatment facilities.

The most dangerous wastewater is produced in the chemical and petrochemical industry. Wastewater of such enterprises is characterized by a complex and variable composition, high content of dissolved impurities, hence biological methods are not always effective. To reduce the wastewater discharge into the sewage system it is proposed to apply the thermal treatment of wastewater with partial or full reuse of purified water in reverse cycle industrial water supply system [16-17]. The purpose of the thermal treatment of wastewater is a termination of its discharge with producing a refined distillate at the same time. The purpose of the study is to consider the use of thermal treatment of wastewater with the subsequent return of treated water to the water supply system of the enterprise and to determine the energy costs for the operation of this scheme [18-22].

Materials and methods It is proposed to consider the process which includes three stages of wastewater treatment with the subsequent return of treated water into the water system (mainly for system replenishment):

Stage 1- removal of mechanical impurities (use of hydrocyclones, mechanical filters, ponds, lagoons, etc.);

Stage 2 - the use of evaporation plant for the concentration of wastewater;

Stage 3 - a dry residue of impurities in the spray dryer, followed by recycling and disposal.

\section{Materials and methods}

Schematic diagram of a zero-discharge water system with heat treatment of wastewater is shown in Fig. 1. 


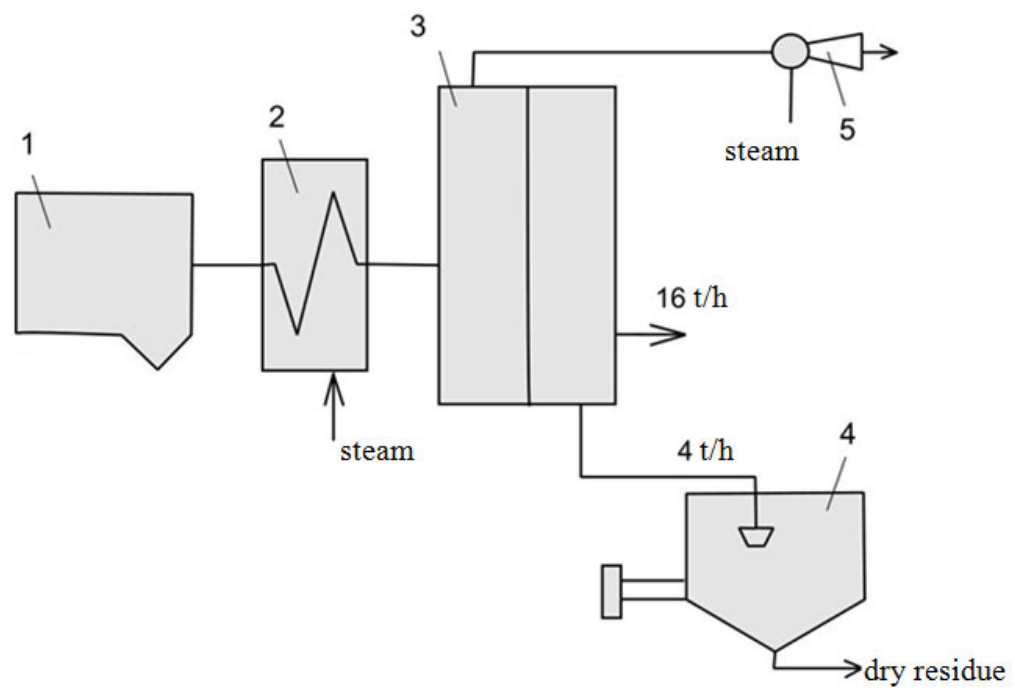

Fig. 1. A zero-discharge water system scheme: 1 - hydrocyclone; 2 - heat exchanger; 3 - instantboiling evaporator; 4 - spray dryer; 5 - ejector.

Wastewater goes into a hydrocyclone, where suspended sludge particles are separated from the aqueous suspension, after that the effluents are heated to a temperature of $90^{\circ} \mathrm{C}$ and fed to the instant-boiling evaporator (IBE). To improve thermal efficiency EIB has multistage configuration with subsequent change of water temperature from 90 to $40^{\circ} \mathrm{C}$.

Each stage of EIB includes following structural elements: the expander, the separator and the condenser (see Fig. 2).

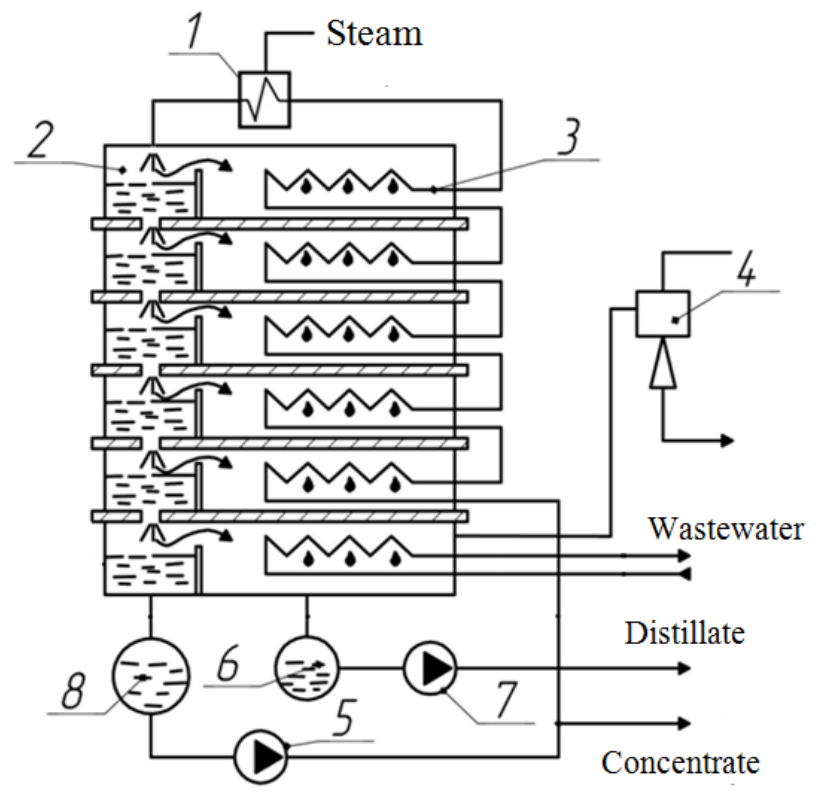

Fig. 2. Instant-boiling evaporator: 1 - heater; 2 - expansion chamber; 3 - condensation chamber; 4 ejector; 5 - circulation pump; 6 - tank for distillate; 7 - distillate pump; 8 - tank for circulating water. 
The main advantages of using instant-boiling evaporators for obtaining of concentrate in the process of industrial wastewater treatment are:

- The absence of moving parts in the EIB, which leads to a safe operation;

- Highly qualified personnel are not required for the EIB maintenance;

- Installation can be operated in automatic mode;

- The quality of the distillate obtained corresponds to the quality of the condensate and it can be reused in the water supply system of industrial enterprise;

- Secure water purification from various types of pollution. Dissolved and suspended substances are removed from the water; mineral and synthetic emulsions are decomposed

- Dimensions of instant-boiling evaporator allow it to be incorporated into the wastewater treatment scheme without high capital costs.

Instant-boiling evaporator comprises a housing, a vertical partition wall with an opening for the passage of steam. The partition wall is tightly coupled to the housing and divides the evaporator into expansion chamber and condensation chamber. Evaporator also comprises pipes for supply and discharge of evaporating water and distillate. Condensation chambers and expansion chambers are equipped with independent bottoms by the number of cameras and tightly connected to the partition wall and the housing of the evaporator. The partition wall is additionally equipped with openings for the passage of steam by the number of expansion chambers and bottoms house cylindrical tubes for the passage of fluid. This allows the performance of the evaporator and reliability of its operation to be improved by eliminating the flow of highly mineralized water to the distillate and preventing contact between the corrosive environment and welded joints on the body.

Boiling does not occur on the heated surface, but in the free volume of the expansion chamber. This, combined with a multistage process of evaporation of the original water, can reduce the risk of scale accumulation in the internal volume of the device. Evaporator blowdown, depending on the quality of the wastewater, takes from 5 to $20 \%$ of plant capacity, and the yield of distillate is from 95 to $80 \%$ of entered volume. Blowdown water (concentrate) is supplied from the EIB to a spray dryer (Fig. 3). The concentrate passes through a high-speed rotary atomizer on the top of the plant and is atomized into fine droplets as a mist. The concentrate is dried instantaneously upon contact with the heated air and is converted into powder. Solids are continuously removed from the bottom of the unit by a screw.

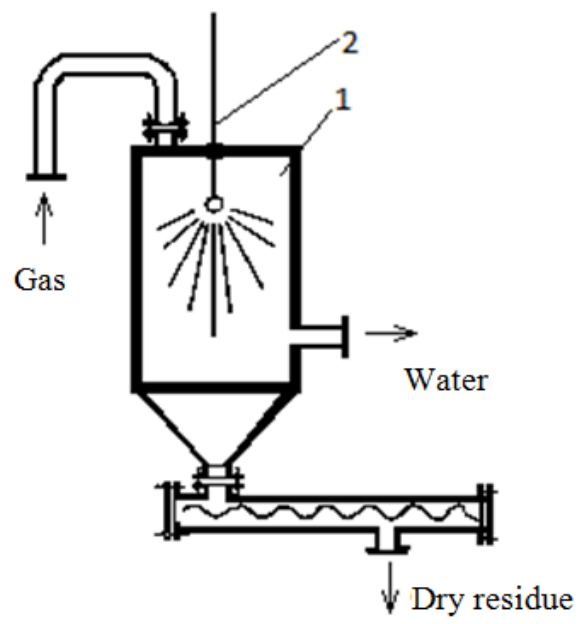

Fig. 3. Spray dryer: 1 - drying chamber; 2 - nozzle. 
Nature of the drying process of materials in an atomized state is that the dispersed liquid droplets or fluid-like mass during its propagation in a confined volume is dewatered due to the difference in partial vapor pressure on the liquid droplets surface and in the environment. Depending on the technological requirements to the material either powder or plastic mass can be obtained in the spray dryer.

In comparison with other methods of drying of liquid and fluid-like materials spray drying has the following advantages:

- Formation of a significant interaction surface between the disperse phase and dispersion medium;

- Short-term process;

- Obtaining of a granulated powder material;

- Mechanization and automation of the drying process.

The spray drying process can be subdivided into three stages: spraying mass; heat and mass transfer between the droplets (particles) and the environment; the allocation of the dried product from the gas stream. Such division of the process is somewhat arbitrary, since it is impossible to identify a clear boundary between the stages due to their imposition of one another.

In the most general case, the spray process is meant to be the liquid jet fragmentation into a large number of droplets and the allocation of these droplets in the volume. Fragmentation of the liquid jet into drops is a very complex process due to a number of external and internal factors. The main external factor is considered to be the impact of the aerodynamic force on the jet surface tending to deform and break it. Internal factors are various kinds of initial perturbations associated with the construction of the sprayer, the quality of its production, the turbulent motion of the liquid in the sprayer and so on. During atomizing the liquid jet is divided into a number of droplets with different diameter.

Methods of obtaining dry residue from the concentrated solution depend on the type of drying facility used.

\section{Results}

The costs of electrical and thermal energy for different amounts of source water were determined.

Table 1. The cost of electrical and thermal energy at different water consumption.

\begin{tabular}{|c|c|c|c|}
\hline $\begin{array}{c}\text { Water } \\
\text { consumption, } \\
\mathrm{t} / \mathrm{h}\end{array}$ & $\begin{array}{c}\text { The amount of } \\
\text { electricity required } \\
\text { for the operation of } \\
\text { the circuit (USD/h) }\end{array}$ & $\begin{array}{c}\text { The amount of heat } \\
\text { required for the operation } \\
\text { of the circuit (USD/h) }\end{array}$ & $\begin{array}{c}\text { Total specific energy } \\
\text { consumption } \\
\text { (USD/h) }\end{array}$ \\
\hline 20 & 37.0 & 55.2 & 92.1 \\
\hline 50 & 105.3 & 128.8 & 234.1 \\
\hline 100 & 351.5 & 248.3 & 599.8 \\
\hline
\end{tabular}

Numerical studies using a complex methodology allowed us to calculate the cost schemes of fuel and energy resources and, based on the results obtained, to calculate the most optimal scheme of water treatment according to the necessary parameters. Based on the results of the calculations, it can be concluded that with an increase in the consumption of wastewater, the energy costs for its treatment increase. When comparing the costs of $20 \mathrm{t} /$ $\mathrm{h}$ and $100 \mathrm{t} / \mathrm{h}$, the cost of electricity increased by 10 times. On thermal energy by 4 times. The total specific energy consumption is 6 times. 


\section{Discussion}

To achieve this goal, theoretical studies were carried out, the most optimal scheme of wastewater treatment of the water supply system for the enterprise was selected at various costs, and a set of numerical studies of water treatment schemes for an industrial enterprise was performed. With the existing method of wastewater treatment of enterprises in Russia, about 50 percent of the energy is spent on maintaining the specified parameters of wastewater treatment for flotation. Our method allows not only to neutralize waste emissions, but also to return up to 80 percent to the recycled water supply system. In the world practice, advanced countries have learned to use up to 95 percent of the waste water of enterprises in their water supply systems. In the future, it is necessary to consider water treatment schemes using less energy-intensive technologies for wastewater treatment. For example, to use devices with immersion burners instead of spray dryers; to consider the possibility of heat recovery with the exhaust gases after the dryers, which will reduce the cost of thermal energy by 23 percent.

\section{Conclusions}

1. The proposed scheme of wastewater treatment allows to reduce the cost of using the industrial water of the enterprise with the use of treated wastewater after treatment for the water supply system for technological needs. The advantage of using these water supply systems is that the water reuse system inside the industrial complex is a highly effective direction in reducing water consumption and wastewater discharge.

2. The advantage of this method of wastewater treatment will reduce the area of existing open reservoirs for wastewater treatment, which will positively affect the ecological situation and reduce the energy load on the operation of treatment facilities.

\section{References}

1. The basis of state policy in the field of environmental development of Russia for the period until 2030 (utv. President of the Russian Federation dated April 30, 2012)

2. V. N. Pokrovskij, E. P. Arakchev, Waste water treatment of thermal power plants (Energy, Moscow, Russia, 256, 1980)

3. RD 153-34.1-42.102-98 Guidelines for the Design of Thermal Separation and Evaporation Plants for the Treatment of Waste Water of TPP and GRES.

4. I. A. Konahina, A. M. Konahin, E. A. Ahmetov, A. I. Fazullina, Improvement of efficiency of condensate collection and cooling water supply systems at large petrochemical enterprises, News of higher educational institutions. Energy issues, 5-6 (2007)

5. E. V. Strizheus, Application of vacuum distillation plants for treatment of industrial effluents, Ecology of production, 2, 56 (2008)

6. REGNUM News Agency. Electronic resource, URL: regnum.ru>news/26965.html (last accessed: 25.04.2021)

7. Business electronic newspaper of Tatarstan «Business online»: Electronic resource. URL: www.business-gazeta.ru/news/315798 (last accessed: 25.04.2021)

8. R. V. Chupin, I. V. Maizel and I. G. Maizel, Comprehensive optimization of water supply and sanitation systems, IOP Conference Series: Materials Science and Engineering 880 (2020) 012040 DOI:10.1088/1757-899X/880/1/012040

9. R. V. Chupin, P. N. Minh, E. A. Gorkov, M. V. Moroz, Indicative and adaptive management of wastewater system improvement (News of universities. Investments. Construction. Real estate) 8, 2(25) (2018) 
10. Yu. Zatsarinnaya, A. Logacheva, K. Suslov, Outlook on the Development of Smart Energy Systems, in Proceedings of the 2020 International Ural Conference on Electrical Power Engineering (UralCon) 9216266, 19-23 (2020) DOI: 10.1109/UralCon49858.2020.9216266

11. Yu. Zatsarinnaya, K. Suslov, A. Danilin 2020 Assessment of the Technical Condition of Turbogenerators by Partial Discharges (Proceedings - 2020 International Ural Conference on Electrical Power Engineering (UralCon) 9216259, 225-229 (2020) DOI: 10.1109/UralCon49858.2020.9216259

12. Yu Zatsarinnaya, A. Logacheva, M. Grigoreva, Electricity in retail markets as a commodity in smart energy systems IOP Conference Series: Materials Science and Engineering, 791 (2020) DOI: 10.1088/1757-899X/791/1/012061

13. Y. N. Zatsarinnaya, A. G. Logacheva, R. N. Gainullin, S. F. Alekseeva, D. I. Amirov, Solution for renewable future, E3S Web of Conferences, 124, 04010 (2019) DOI: 10.1051/e3sconf/201912404010

14. J. N. Zatsarinnaya, A. G. Logacheva, A. A. Solovyova, Analysis of thermodynamic efficiency of the fuel preparation systems with an intermediate hopper at thermal power plan, in proceedings of the International Scientific and Practical Conference: Water Power Energy Forum, 2018 DOI:10.1088/1755-1315/288/1/012130

15. A. A. Akhmetov, R. V. Akhmetova, Development of water saving technology for water supply system of industrial enterprises, IOP Conference Series: Materials Science and Engineering, 134(1),012001 (2016) DOI:10.1088/1757-899X/134/1/012001

16. S. Uchida, Low temperature absorption refrigeration machine with water-LiBr mixed refrigerant, Japan, Refrigeration, 81, 946, 618-621 (2016)

17. Z. Y. Xu, Absorption refrigeration cycles: Categorized based on the cycle construction, Int. J. of Refrigeration, 62, 114-136 (2016) DOI: 10.1016/j.ijrefrig.2015.10.007

18. R. Z. Wang, Experimental evaluation of avariable effect LiBr-water absorption chiller designed for high-efficient solar cooling system, Int. J. of Refrigeration, 69, 135-143 (2017) DOI: 10.1016/j.ijrefrig.2015.07.019

19. S. Pedersen, $18 \mathrm{MW}$ heat pump plant utilising low-potential waste water heat,(Thermal pumps thermal pumps, 1, 36-37 (2018)

20. R. V. Chupin, O. G. Primin, Optimization of the sewerage systems scheme of cities and populated areas, IOP Conference Series: Materials Science and Engineering 667 (2019) 012018 DOI:10.1088/1757-899X/667/1/012018

21. R. V. Chupin, N. M. Pham, Optimization of developing district water supply systems taking into account variability of perspective water consumption, IOP Conference Series: Materials Science and Engineering, 667 (2019) 012017 DOI: 10.1088/1757899X/667/1/012017

22. R. R. Rotach, E. A. Akhmetov, S. O. Gaponenko, Definition of an optimum method of water preparation for needs of the enterprises, IOP Conference Series: Earth and Environmental Science, 288(1), 012025 (2019) DOI: 10.1088/1755-1315/288/1/012025 